\title{
Lump Solutions to the (3+1)-Dimensional Generalized B-Type Kadomtsev-Petviashvili Equation
}

\author{
Xifang Cao \\ School of Mathematical Sciences, Yangzhou University, China \\ Correspondence should be addressed to Xifang Cao; xfcao@yzu.edu.cn
}

Received 13 August 2018; Revised 26 September 2018; Accepted 11 October 2018; Published 1 November 2018

Academic Editor: Antonio Scarfone

Copyright (C) 2018 Xifang Cao. This is an open access article distributed under the Creative Commons Attribution License, which permits unrestricted use, distribution, and reproduction in any medium, provided the original work is properly cited.

This paper is devoted to the study of lump solutions to the (3+1)-dimensional generalized B-type Kadomtsev-Petviashvili equation. First we use a direct method to construct a class of exact solutions which contain six arbitrary real constants. Then we use these solutions to generate lump solutions with four real parameters. We also determine the amplitude and velocity of these lumps.

\section{Introduction}

Lump is a type of localized rational solutions. Lump solutions for the (2+1)-dimensional Kadomtsev-Petviashvili I equation

$$
\left(u_{t}+6 u u_{x}+u_{x x x}\right)_{x}-u_{y y}=0
$$

were first obtained by Manakov et al. [1]. Then Satsuma and Ablowitz [2] constructed more lump solutions by taking a long wave limit of the corresponding soliton solutions. More recently, by use of the bilinear form, $\mathrm{Ma}$ [3] generalized the results of $[1,2]$ and got a larger class of lump solutions. Over the past few decades, lump has been an active area in the study of nonlinear evolution equations. They can be used to describe nonlinear patterns in plasma [4], in optical media [5], in the Bose-Einstein condensate [6], and so forth. Lump solutions have been obtained for some other equations, such as the Ishimori equation [7], the Jimbo-Miwa equation [8], and the Sawada-Kotera equation [9].

Recently much literature is devoted to the study of $(3+1)$ dimensional B-type Kadomtsev-Petviashvili (BKP) equation

$$
u_{y t}+3 u_{x z}-3 u_{x} u_{x y}-3 u_{x x} u_{y}-u_{x x x y}=0 \text {, }
$$

which can be used to model fluid dynamics, plasma physics, and weakly dispersive media $[10,11]$. Various methods have been applied to (2) to construct its soliton and multiple wave solutions [12-14]. In this paper, we develop another method to construct its lump solutions. We first use a direct method to obtain a class of exact solutions which contain six arbitrary real constants (see Theorem 1). Then we show that the limits of these solutions can generate lump solutions (see Theorem 4). We also determine the amplitude and velocity of these lumps.

\section{Exact Solutions}

In this section, we seek exact solutions to the $(3+1)$-dimensional BKP equation (2) in the following form:

$$
u(x, y, z, t)=2(\ln f)_{x},
$$

where

$$
f(x, y, z, t)=\exp (-\eta)+m \cos \xi+n \exp \eta
$$

$\eta=p(x+q y+r z), \xi=p(a x+b y+c z+d t)$, and $m, n, p, q, r, a, b$, $c, d$ are real constants to be determined. Substituting (3) into the left hand side of (2) and then factoring we get the following three determining equations:

$$
\begin{aligned}
& 6 a c-3 r+b d+3 a^{2} r+p^{2} q+4 a^{3} b p^{2}-6 a^{2} p^{2} q \\
& \quad+a^{4} p^{2} q+a d q-4 a b p^{2}=0 \\
& 3 c+6 a r+d q-3 a^{2} c-b p^{2}-4 a p^{2} q+6 a^{2} b p^{2}-a^{4} b p^{2} \\
& \quad+4 a^{3} p^{2} q-a b d=0
\end{aligned}
$$




$$
\begin{aligned}
& m^{2}\left(3 r+18 a c+3 b d+9 a^{2} r-p^{2} q+24 a^{3} b p^{2}\right. \\
& \left.\quad-6 a^{2} p^{2} q+3 a^{4} p^{2} q+3 a d q\right)+16 n\left(4 p^{2} q-3 r\right) \\
& \quad=0 .
\end{aligned}
$$

Solving for $r, c$ from (5) and (6) gives

$$
r=\frac{p^{2} q+b d-a^{4} p^{2} q-2 a^{3} b p^{2}-2 a b p^{2}-a d q}{3\left(a^{2}+1\right)}
$$

and

$$
c=\frac{b p^{2}+2 a^{3} p^{2} q+2 a p^{2} q-a^{4} b p^{2}-a b d-d q}{3\left(a^{2}+1\right)} .
$$

Substituting (8) and (9) into (7) yields

$$
\begin{aligned}
n= & -\frac{3 b a^{5} p^{2}+2 q a^{4} p^{2}+4 b a^{3} p^{2}+2 q a^{2} p^{2}+b a p^{2}-d q a+b d}{4\left(q a^{4} p^{2}+2 b a^{3} p^{2}+4 q a^{2} p^{2}+2 b a p^{2}+3 q p^{2}+d q a-b d\right)} \\
& \cdot m^{2} .
\end{aligned}
$$

Therefore, we have the following.

Theorem 1. The (3+1)-dimensional BKP equation (2) admits the following exact solutions:

$$
\begin{aligned}
& u(x, y, z, t)=2(\ln f)_{x}, \\
& f(x, y, z, t)=\exp (-\eta)+m \cos \xi+n \exp \eta
\end{aligned}
$$

where $\eta=p(x+q y+r z), \xi=p(a x+b y+c z+d t), m, p, q, a, b, d$ are arbitrary real constants, and $r, c, n$ are given by (8), (9), and (10), respectively.

\section{Lump Solutions}

In this section, we use (11) to construct lump solutions to the (3+1)-dimensional BKP equation (2). We first give some examples and then give a general result.

Example 1. Let $m=-2, q=1, a=0, b=1, d=1$. Then (11) becomes

$$
\begin{aligned}
& u(x, y, z, t)=2(\ln f)_{x} \\
& f(x, y, z, t)=\exp (-\eta)-2 \cos \xi+\frac{1}{1-3 p^{2}} \exp \eta
\end{aligned}
$$

where $\eta=p\left(x+y+\left(\left(1+p^{2}\right) / 3\right) z\right), \xi=p\left(y+\left(\left(p^{2}-1\right) / 3\right) z+t\right)$. As $p \longrightarrow 0$, the limit of (12) gives a lump solution to (2):

$$
U(x, y, z, t)=\lim _{p \rightarrow 0} u(x, y, z, t)=\frac{4 X}{3+X^{2}+Y^{2}},
$$

where

$$
\begin{aligned}
& X=x+y+\frac{z}{3} \\
& Y=y-\frac{z}{3}+t .
\end{aligned}
$$

The lump (13) attains its maximum value $2 / \sqrt{3}$ at $(X, Y)=$ $(\sqrt{3}, 0)$ and minimum value $-2 / \sqrt{3}$ at $(X, Y)=(-\sqrt{3}, 0)$. So its amplitude is $2 / \sqrt{3}$. The profiles of the lump (13) in the $(X, Y)$-space are given in Figure 1 .

From the point of view of the $(x, y, z)$-space, at each time $t$, the lump (13) attains its extreme values at the lines

$$
\begin{gathered}
x+y+\frac{z}{3}= \pm \sqrt{3}, \\
-3 y+z=3 t .
\end{gathered}
$$

Note that the planes $x+y+z / 3= \pm \sqrt{3}$ are fixed (do not depend on $t$ ), while the plane $-3 y+z=3 t$ moves along its normal vector $\mathbf{N}=(1 / \sqrt{10})(0,-3,1)$ with the speed $3 / \sqrt{10}$. Therefore the velocity of the moving lines (15) is

$$
\mathbf{V}=\left(V_{x}, V_{y}, V_{z}\right)=\frac{63}{5 \sqrt{266}}(8,-11,9) \text {. }
$$

Example 2. Let $m=-2, q=0, a=1, b=1, d=1$. Then (11) becomes

$$
\begin{aligned}
& u(x, y, z, t)=2(\ln f)_{x} \\
& f(x, y, z, t)=\exp (-\eta)-2 \cos \xi+\frac{1+8 p^{2}}{1-4 p^{2}} \exp \eta
\end{aligned}
$$

where $\eta=p\left(x+\left(\left(1-4 p^{2}\right) / 6\right) z\right), \xi=p(x+y-(1 / 6) z+t)$. As $p \longrightarrow 0$, the limit of (17) gives a lump solution to (2):

$$
U(x, y, z, t)=\lim _{p \rightarrow 0} u(x, y, z, t)=\frac{4(X+Y)}{12+X^{2}+Y^{2}},
$$

where

$$
\begin{aligned}
& X=x+\frac{z}{6} \\
& Y=x+y-\frac{z}{6}+t
\end{aligned}
$$

The lump (18) attains its maximum value $\sqrt{6} / 3$ at $(X, Y)=$ $(\sqrt{6}, \sqrt{6})$ and minimum value $-\sqrt{6} / 3$ at $(X, Y)=(-\sqrt{6}$, $-\sqrt{6})$. So the amplitude of the lump (18) is $\sqrt{6} / 3$. The profiles of the lump (18) in the $(X, Y)$-space are given in Figure 2.

From the point of view of the $(x, y, z)$-space, at each time $t$, the lump (18) attains its extreme values at the lines

$$
\begin{array}{r}
x+\frac{z}{6}= \pm \sqrt{6}, \\
x+y-\frac{z}{6}+t= \pm \sqrt{6},
\end{array}
$$

which move with the velocity

$$
\mathbf{V}=\left(V_{x}, V_{y}, V_{z}\right)=\frac{1476}{73 \sqrt{1517}}(-2,-37,12) .
$$

Example 3. Let $m=-2, q=1, a=-1, b=0, d=1$. Then (11) becomes

$$
\begin{aligned}
& u(x, y, z, t)=2(\ln f)_{x}, \\
& f(x, y, z, t)=\exp (-\eta)-2 \cos \xi+\frac{1+4 p^{2}}{1-8 p^{2}} \exp \eta
\end{aligned}
$$



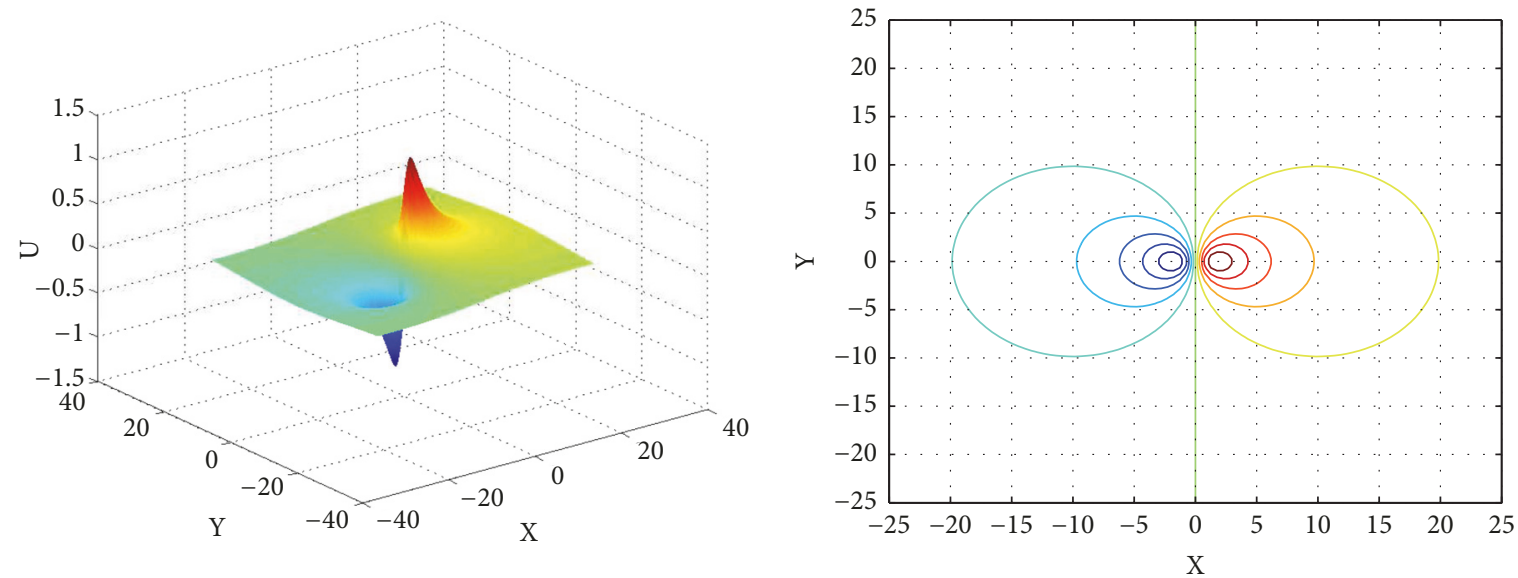

FIGURE 1: The 3D plot of lump (13) and the corresponding contour plot.
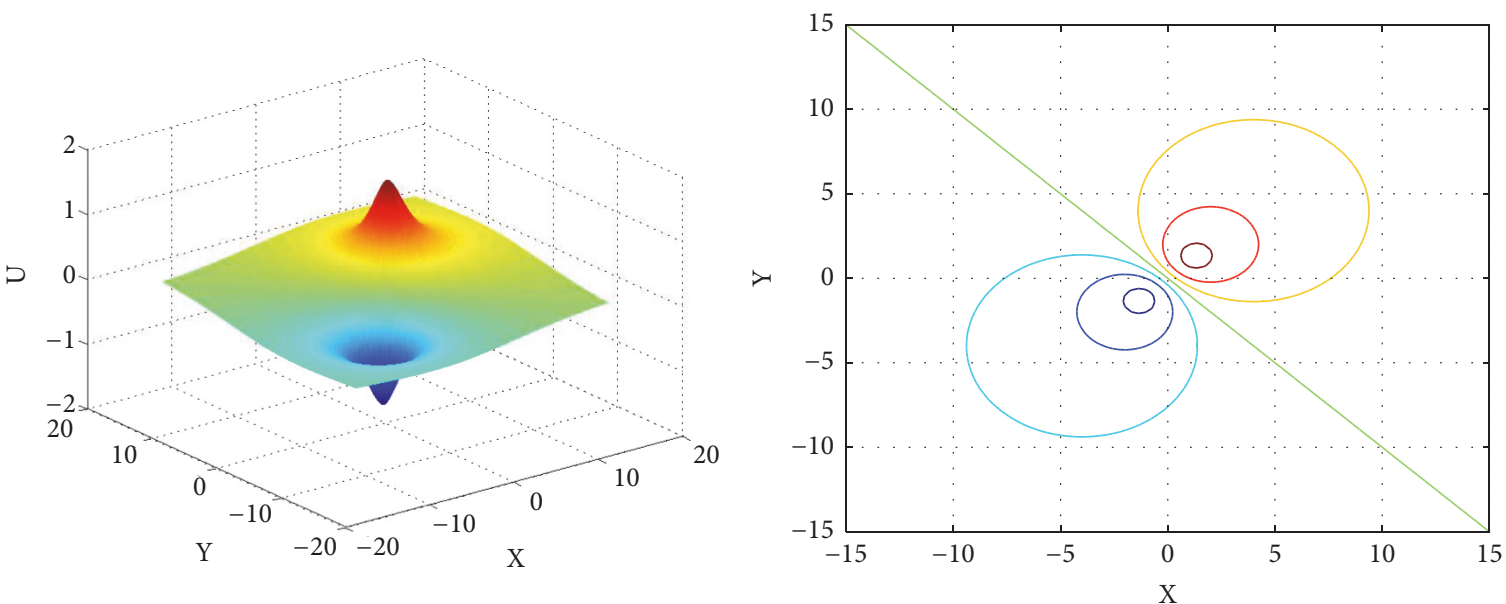

FIgURE 2: The 3D plot of lump (18) and the corresponding contour plot.

where $\eta=p(x+y+(1 / 6) z), \xi=p\left(-x+\left(\left(4 p^{2}-1\right) / 6\right) z+t\right)$. As $p \longrightarrow 0$, the limit of (22) gives a lump solution to (2):

$$
U(x, y, z, t)=\lim _{p \rightarrow 0} u(x, y, z, t)=\frac{4(X-Y)}{12+X^{2}+Y^{2}},
$$

where

$$
\begin{aligned}
& X=x+y+\frac{z}{6}, \\
& Y=-x-\frac{z}{6}+t .
\end{aligned}
$$

The lump (23) attains its maximum value $\sqrt{6} / 3$ at $(X, Y)=(\sqrt{6},-\sqrt{6})$ and minimum value $-\sqrt{6} / 3$ at $(X, Y)=$ $(-\sqrt{6}, \sqrt{6})$. So the amplitude of the lump $(23)$ is $\sqrt{6} / 3$. The profiles of the lump (23) in the $(X, Y)$-space are given in Figure 3.

From the point of view of the $(x, y, z)$-space, at each time $t$, the lump (23) attains its extreme values at the lines

$$
\begin{aligned}
& x+y+\frac{z}{6}= \pm \sqrt{6} \\
& -x-\frac{z}{6}+t=\mp \sqrt{6}
\end{aligned}
$$

which move with the velocity

$$
\mathbf{V}=\left(V_{x}, V_{y}, V_{z}\right)=\frac{1332}{37 \sqrt{2701}}(36,-37,6) \text {. }
$$

In general, for arbitrary real constants $q, a, b, d$, let $m=$ -2 . Then as $p \longrightarrow 0$, the limit of (11) yields the following result.

Theorem 4. The (3+1)-dimensional BKP equation (2) admits the following rational solutions:

$$
U(x, y, t)=4 \frac{X+a Y}{A+X^{2}+Y^{2}}
$$



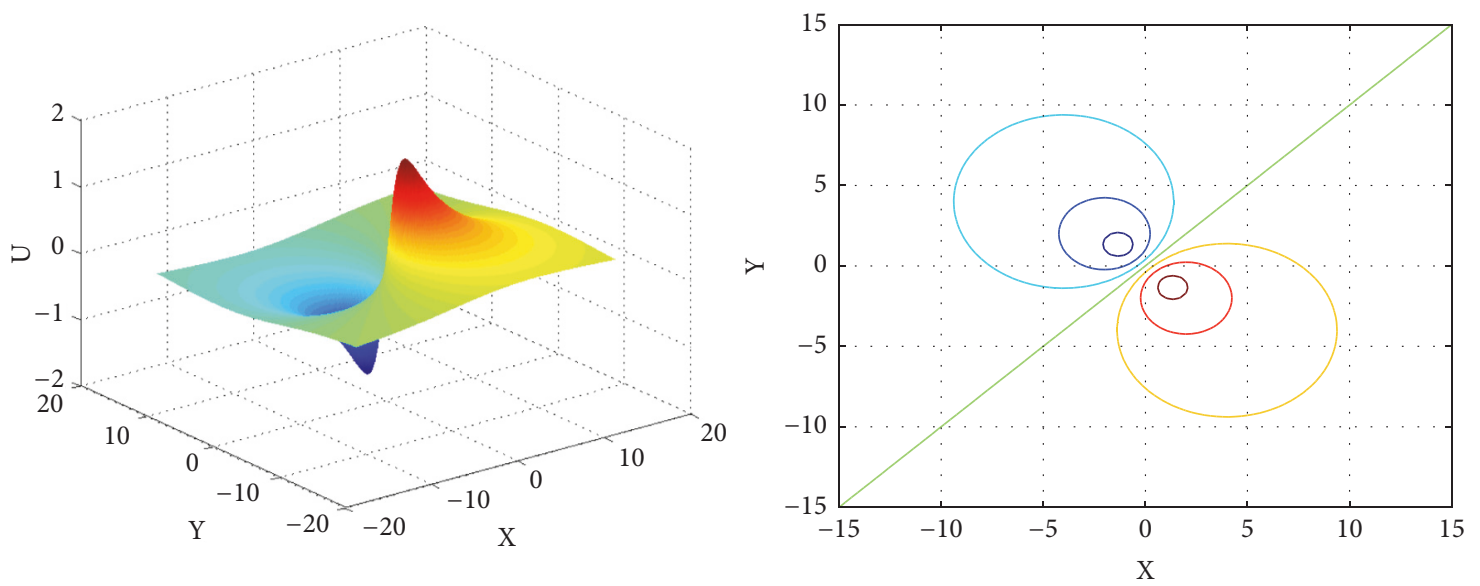

Figure 3: The 3D plot of lump (23) and the corresponding contour plot.

where

$$
\begin{aligned}
& A=\frac{3\left(a^{2}+1\right)^{2}(q+a b)}{d(b-a q)}, \\
& X=x+q y-\frac{d(a q-b)}{3\left(a^{2}+1\right)} z, \\
& Y=a x+b y-\frac{d(q+a b)}{3\left(a^{2}+1\right)} z+d t,
\end{aligned}
$$

and $q, a, b, d$ are arbitrary real constants.

Remark 5. If the parameters $q, a, b, d$ satisfy the following condition,

$$
\frac{q+a b}{d(b-a q)}>0
$$

then (27) are lump solutions.

For given parameters $q, a, b, d$ satisfying condition (31), the lump (27) attains its extreme values $\pm 2 \sqrt{d(b-a q) / 3\left(a^{2}+1\right)(q+a b)}$ at the lines

$$
\begin{aligned}
& x+q y-\frac{d(a q-b)}{3\left(a^{2}+1\right)} z= \pm \sqrt{\frac{3\left(a^{2}+1\right)(q+a b)}{d(b-a q)},} \\
& a x+b y-\frac{d(q+a b)}{3\left(a^{2}+1\right)} z+d t \\
& = \pm a \sqrt{\frac{3\left(a^{2}+1\right)(q+a b)}{d(b-a q)}}
\end{aligned}
$$

in the $(x, y, z)$-space. Therefore the amplitude of the lump (27) is

$$
U_{\text {amplitude }}=2 \sqrt{\frac{d(b-a q)}{3\left(a^{2}+1\right)(q+a b)}} .
$$

The velocity $\mathbf{V}=\left(V_{x}, V_{y}, V_{z}\right)$ of the lump (27) in the $(x, y, z)$ space can also be determined. Here we omit the details.

\section{Discussion}

The (3+1)-dimensional generalized B-type Kadomtsev-Petviashvili equation is the Jimbo-Miwa equation. If we replace $t$ with $-t / 2$, then (2) becomes

$$
u_{x x x y}+3 u_{y} u_{x x}+3 u_{x} u_{x y}+2 u_{y t}-3 u_{x z}=0
$$

Based on the bilinear form of (34), Yang and Ma [8] construct ten classes of its lump solutions in the form

$$
u=2(\ln f)_{x}, \quad f=g^{2}+h^{2}+a_{11},
$$

where $g=a_{1} x+a_{2} y+a_{3} z+a_{4} t+a_{5}, h=a_{6} x+a_{7} y+a_{8} z+$ $a_{9} t+a_{10}$, and $a_{i}(i=1, \ldots, 11)$ are some constants.

In this section, we point out that up to the invariance after a translation and a Galilean transformation, all the solutions obtained in [8] can be simplified and be rewritten in a unified form of (27). This is because, firstly, the parameters $a_{5}$ and $a_{10}$ can be set zero by the translation $(x, y, z, t) \longrightarrow\left(x^{\prime}, y^{\prime}, z^{\prime}, t^{\prime}\right)$ defined by

$$
\begin{aligned}
& x^{\prime}=x+x_{0}, \\
& y^{\prime}=y+y_{0} \\
& z^{\prime}=z+z_{0} \\
& t^{\prime}=t+t_{0}
\end{aligned}
$$

where $x_{0}, y_{0}, z_{0}, t_{0}$ are some constants; secondly, since the parameters $a_{1}$ and $a_{6}$ can not be zero simultaneously, without loss of generality, we may assume $a_{1} \neq 0$, and then from the form of (35), we can set $a_{1}=1$; thirdly, since (34) is invariant under the Galilean transformation $(x, y, z, t) \longrightarrow$ $\left(x^{\prime}, y^{\prime}, z^{\prime}, t^{\prime}\right)$ given by 


$$
\begin{aligned}
& x^{\prime}=x+3 \lambda t, \\
& y^{\prime}=y+2 \lambda z, \\
& z^{\prime}=z, \\
& t^{\prime}=t,
\end{aligned}
$$

where $\lambda$ is an arbitrary real number, we can set $a_{4}=0$. Then under the condition

$$
\begin{gathered}
a_{1}=1, \\
a_{4}=0, \\
a_{5}=0, \\
a_{10}=0,
\end{gathered}
$$

$g$ and $h$ are of the forms of (29) and (30), and a direct calculation shows that each class of solutions in [8] can be rewritten in the unified form of (27).

From the mathematical point of view, the lump solutions (27) are limits of the exact solutions (11) as the parameter $p$ approaches zero. It is worth studying the physical meaning of this limit.

\section{Data Availability}

No data were used to support this study.

\section{Conflicts of Interest}

The author declares that there are no conflicts of interest.

\section{Acknowledgments}

This work is supported by the Natural Science Foundation of Jiangsu Province through BK20151304.

\section{References}

[1] S. V. Manakov, V. E. Zakharov, L. A. Bordag, A. R. Its, and V. B. Matveev, "Two-dimensional solitons of the Kadomtsev-Petviashvili equation and their interaction," Physics Letters A, vol. 63, no. 3, pp. 205-206, 1977.

[2] J. Satsuma and M. J. Ablowitz, "Two-dimensional lumps in nonlinear dispersive systems," Journal of Mathematical Physics, vol. 20, no. 7, pp. 1496-1503, 1979.

[3] W.X. Ma, "Lump solutions to the Kadomtsev-Petviashvili equation," Physics Letters A, vol. 379, no. 36, pp. 1975-1978, 2015.

[4] M. J. Ablowitz and H. Segur, Solitons and the Inverse Scattering Transform, SIAM, Philadelphia, PA, USA, 1981.

[5] D. E. Pelinovsky, Y. A. Stepanyants, and Y. S. Kivshar, "Selffocusing of plane dark solitons in nonlinear defocusing media," Physical Review E: Statistical, Nonlinear, and Soft Matter Physics, vol. 51, no. 5, pp. 5016-5026, 1995.

[6] V. A. Mironov, A. I. Smirnov, and L. A. Smirnov, "Structure of vortex shedding past potential barriers moving in a BoseEinstein condensate," Journal of Experimental and Theoretical Physics, vol. 110, no. 5, pp. 877-889, 2010.
[7] K. Imai, "Dromion and lump solutions of the Ishimori-I equation," Progress of Theoretical Physics, vol. 98, no. 5, pp. 1013-1023, 1997.

[8] J. Y. Yang and W. X. Ma, "Abundant lump-type solutions of the Jimbo-Miwa equation in (3+1)-dimensions," Computers and Mathematics with Applications, vol. 73, no. 2, pp. 220-225, 2017.

[9] L. L. Huang and Y. Chen, "Lump solutions and interaction phenomenon for $(2+1)$-dimensional Sawada-Kotera equation," Communications in Theoretical Physics, vol. 67, no. 5, pp. 473478, 2017.

[10] E. Date, M. Jimbo, M. Kashiwara, and T. Miwa, "Transformation groups for soliton equations VI-KP hierarchies of orthogonal and symplectic type," Journal of the Physical Society of Japan, vol. 50, no. 11, pp. 3813-3818, 1981.

[11] E. Date, M. Jimbo, M. Kashiwara, and T. Miwa, "Transformation groups for soliton equations: IV. A new hierarchy of soliton equations of KP-type," Physica D: Nonlinear Phenomena, vol. 4, no. 3, pp. 343-365, 1982.

[12] W. X. Ma and E. G. Fan, "Linear superposition principle applying to Hirota bilinear equations," Computers and Mathematics with Applications, vol. 61, pp. 950-959, 2011.

[13] W. X. Ma and Z. Zhu, "Solving the $(3+1)$-dimensional generalized KP and BKP equations by the multiple exp-function algorithm," Applied Mathematics and Computation, vol. 218, no. 24, pp. 11871-11879, 2012.

[14] S. Liu, Y. Ding, and J. G. Liu, "A class of exact solutions of (3+1)dimensional generalized B-type Kadomtsev-Petviashvili equation," International Journal of Nonlinear Sciences and Numerical Simulation, vol. 18, no. 2, pp. 137-144, 2017. 


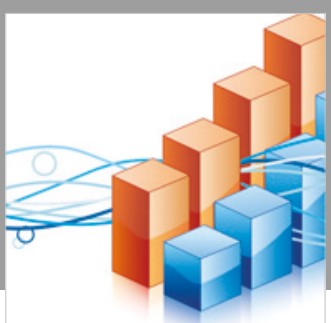

Advances in

Operations Research

\section{-n-m}
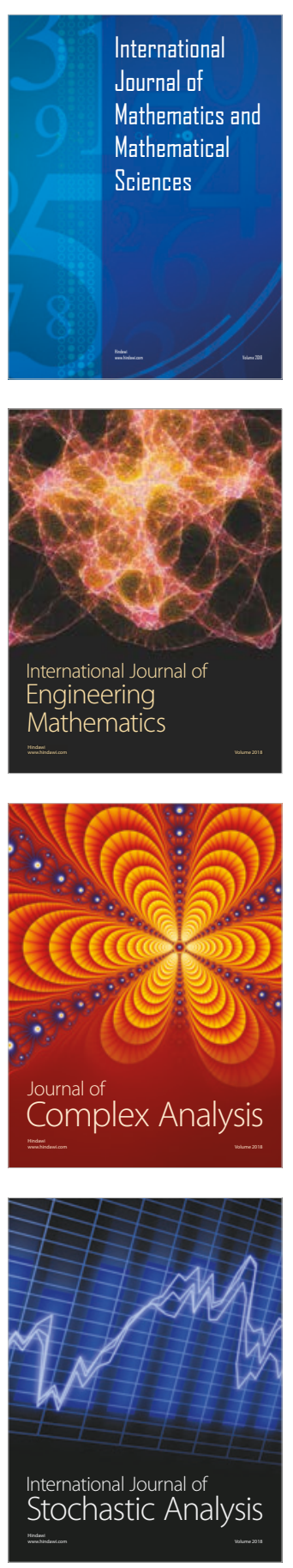
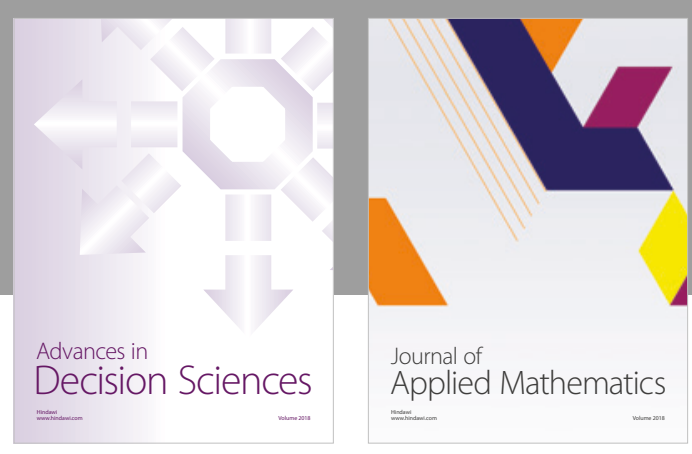

Journal of

Applied Mathematics
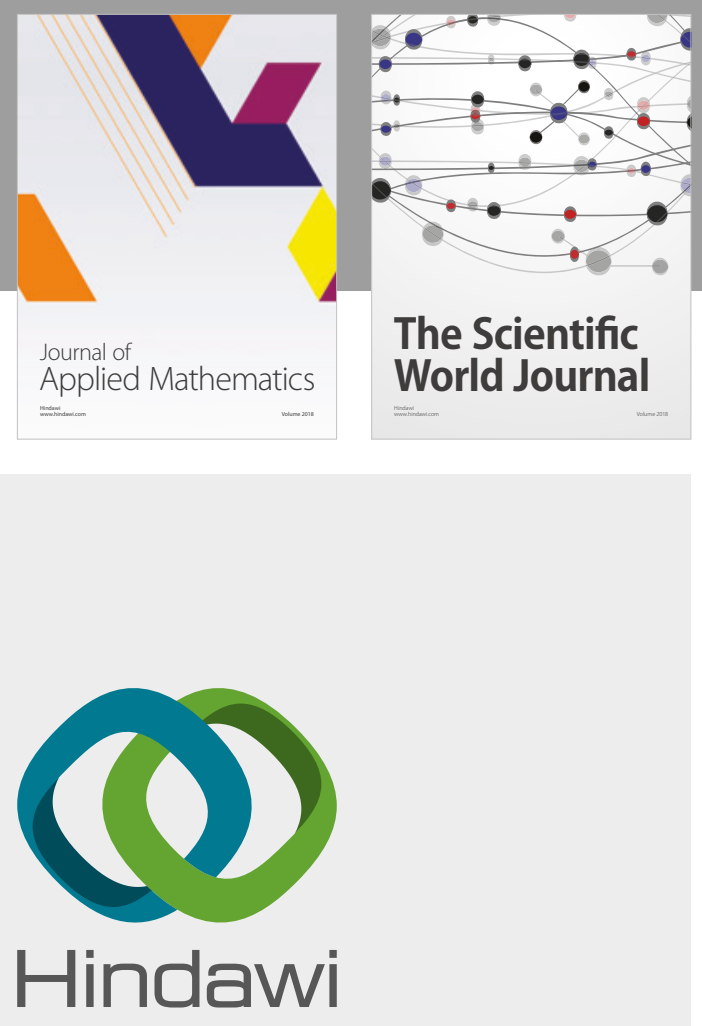

Submit your manuscripts at

www.hindawi.com

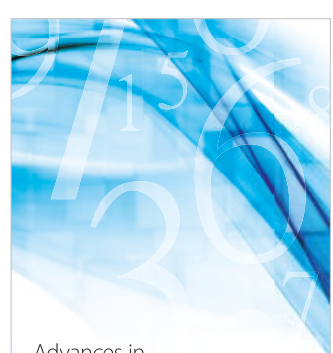

Advances in
Numerical Analysis
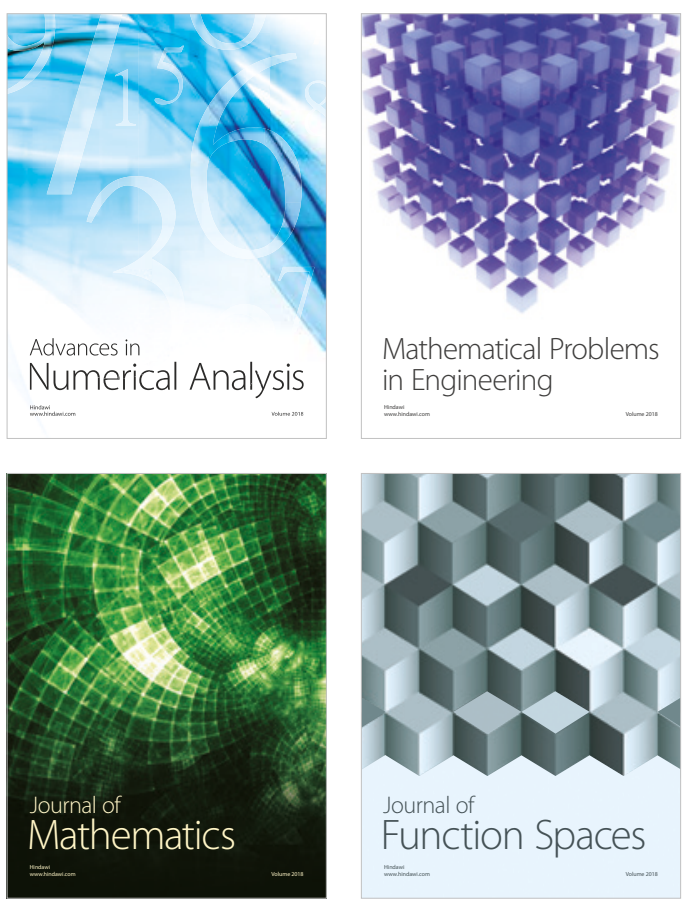

Mathematical Problems in Engineering

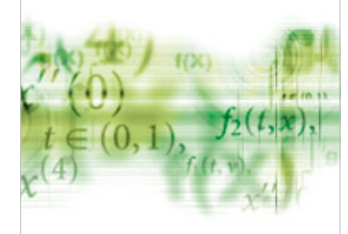

International Journal of

Differential Equations

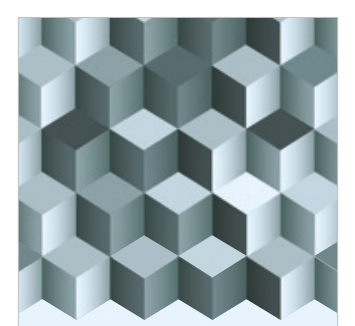

Journal of

Function Spaces

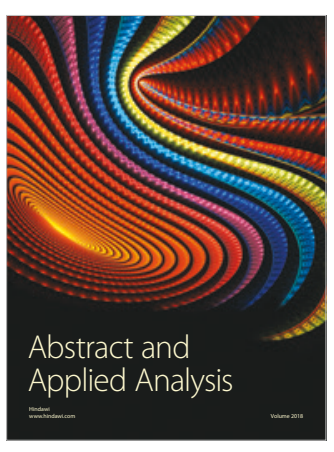

The Scientific

World Journal

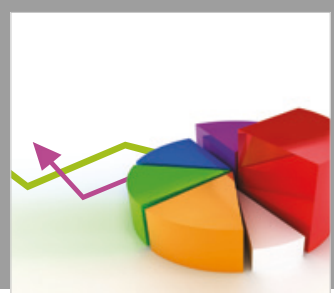

Journal of

Probability and Statistics
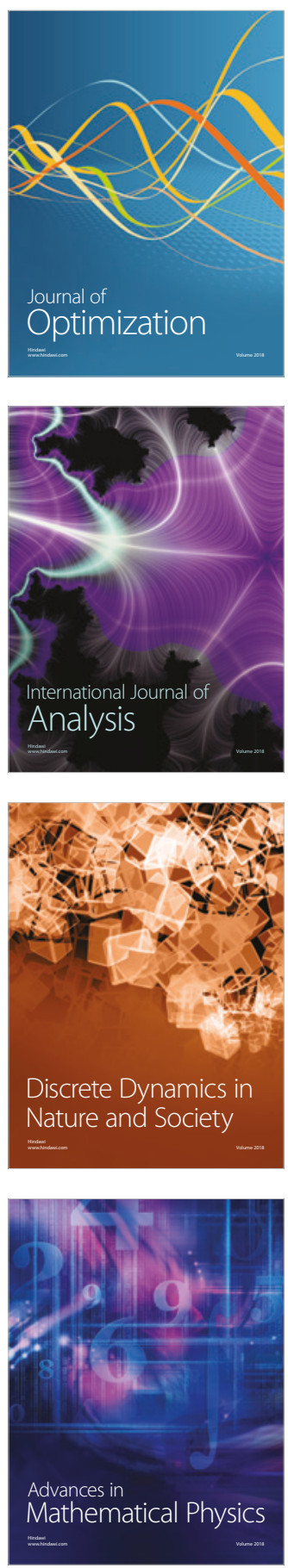